\title{
SUPPORT FOR COMMUNICATION WITH DEAF AND DUMB PATIENTS VIA FEW-SHOT MACHINE LEARNING
}

\author{
Grigorii Shovkoplias, Mark Tkachenko, Arip Asadulaev, Olga Alekseeva, Natalia Dobrenko, \\ Daniil Kazantsev, Alexandra Vatian, Anatoly Shalyto and Natalia Gusarova \\ ITMO University, Saint Petersburg 197101, Russia
}

\begin{abstract}
Improving healthcare quality and patient safety for patients with disabilities is one of the most important goals of e-Health. A large percentage of these patients are persons with disabling hearing loss, i.e. partially or completely deaf and dumb. In our work, we discuss the opportunity of fast Sign Language processing having only a small number of examples. We investigated the possibility of classifying datasets with an extremely small number of samples of electromyograms of deaf and dumb gestures using few-shots learning methods. Several such methods have been considered - Matching Networks, Model-Agnostic Meta-Learning, and Prototypical Networks. The developed methodology makes it possible to train electromyogram classifiers using an extremely small amount of data for other deaf and dumb sign languages. To do this, it is enough to collect a small dataset for re- or additional training of these models for the classification of another language, which is easy to accomplish in practice by means of a small session of recording the gestures of several deaf-mute people - speakers of a particular sign language. In our study, we selected three main few-shot learning approaches and compared them on small sign language dataset. Based on our results, one can choose the best models and their modifications to adapt to the practice task.
\end{abstract}

\section{KEYWORDS}

Sign Language, Machine Learning, Marker Method, Neural Networks

\section{INTRODUCTION}

Improving healthcare quality and patient safety for patients with disabilities is one of the most important goals of e-Health. A large percentage of these patients are persons with disabling hearing loss, i.e. partially or completely deaf and dumb. According to World Health Organization, they represent more than 5\% of the world's population - 466 million people (432 million adults and 34 million children). The main means of communication for the deaf and hearing-impaired people is sign language, which, apparently, most medical personnel do not know. Translation from sign language into a usual, sound language, requires a human sign language interpreter; this is not only expensive, but also slows down the pace of communication, which is especially important while providing emergency medical care. In addition, such an approach fundamentally violates medical secrecy and the requirements for personalizing medical information. Therefore, a promising way is the development and widespread introduction into medical practice of IT tools for sign language processing, that is, computer sign language interpretation systems that convert a sequence of gestures (sign speech) into text in a spoken language.

There are two ways to recognize gestures using technical means: markerless and marker (see review papers [Agrawal, Bragg, Kamal]). The markerless method realizes recognition of human gestures using video cameras and infrared transmitters that allow tracking movements, with subsequent processing of the video stream. The marker system assumes that a person is put on sensors that take into account the movement of muscles and points on the person's body - special gloves, devices on the wrists, bracelets, etc.

The markerless method involves the use of a stationary set of equipment and a high complexity of video stream processing, which makes such technologies expensive and inactive. In addition, very few of the published markless developments [Ee, Guo, Huang] are aimed at recognizing a continuous flow of sign 
language, rather than individual gestures. Today they do not go beyond the scope of research and development and are hardly promising for mass use in e-Health systems.

The marker method is fundamentally simpler, and many companies are working in this direction, already declaring their products as commercial [O'Connor, Song, Zhou]. In medical practice, it is enough to put on the patient a set of equipment for taking EMG in the forearm area [Myo]. The patient (a native speaker of sign language) spells the words in his usual way, each gesture is classified in accordance with our proposed model. The resulting sequential set of letters is converted into words and then displayed on the screen of the doctor's mobile phone or converted into audio speech. However, in this case, too, there are signal processing difficulties associated with the need to take into account the current context when processing specific tokens, i.e. with the implementation of the sequence-to-sequence transformation, as well as with low noise immunity of the received signal. As a result, the quality of real computer sign language interpretation systems remains low, which is especially important for organizing full-fledged communication in the provision of emergency medical care.

Artificial Intelligence (AI) and machine learning technologies, in particular Deep Learning (DL), are the mainstay for the problems of this kind. But attempts to use AI tools in the situation discussed come across, in addition, small volumes of available datasets for training, especially for sign languages other than English Sign Language (SL), as is the case in most underdeveloped and developing countries around the world.

In our work, we discuss the opportunity of fast SL processing having only a small number of examples. There are several methods for working with small amounts of data. In particular, in solving the problem of small datasets in AI, the few-shot approach [Wang] is considered to be promising. In our study, we selected three main few-shot learning approaches and compared them on small SL dataset. Based on our results, one can choose the best models and their modifications to adapt to the practice task.

In our work in Section 2 we provide an overview of few-shot learning techniques and their shortcomings for on-line marker SL recognition, in Section 3 we present our problem-oriented modification of few-shot learning techniques. Section 4 presents the results of our experimental verification of the proposed modification in relation to the three most advanced implementations of the fu-shot approach. The Conclusion briefly presents the results and further directions of our work.

\section{BACKGROUND}

Human intelligence can recognize new forms of a viewed object from a few examples leveraging the knowledge obtained from the previous learning experience. For the existing architectures of DL, it is a complex problem to extract previous knowledge from the "memory" and use them in the new task. DL methods often require massive data for discriminative tasks. In most models, each task is treated independently and the parameters learn from the ground up without integrating task-specific prior knowledge.

Methods that are aimed to incorporate previous knowledge and address low data problems are named few-shot learning. The focus of recent few-shot learning [Wang] research has been on the development of learning methods that can quickly adapt to unseen tasks with small amounts of data and low computational cost. To do this, there are two main approaches - Optimization-Based and Metric-Based.

Optimization-based few-shot learning frameworks use gradient descent-based methods as the optimization process in the meta-learning. Optimization based-methods propose to share knowledge across all tasks. In order to obtain optimal initial stage model-agnostic meta-learning method is proposed [Finn] learning to predict initial parameters of convolutional neural network for each task. Some methods are proposed to learn the task-specific knowledge too [Lee]. The main drawback of optimization-based approaches is a high degree of consistent and the ability to obtain an optimal initial optimization state. Besides, optimization-based methods are computationally expensive and require a large number of similar tasks for optimal performance.

Metric-based methods for few-shot learning are based on the similarity between samples. The trained model can classify samples based on the distance between them. The basic metric-based approach is a Siamese Network [Koch] where two neural networks with the same architecture are trained to learn the similarity between support set and query sample through L1-distance. Different metrics can be used to calculate the similarity between embeddings, for example cosine distance, squared Euclidean distance, L1-distance. Compared with other approaches, metric-based approaches are efficient for few-shot learning and work faster as they do not require the backpropagation process. The advanced technique for the metric-based few-shot 
learning is prototypical networks [Snell] that compute squared Euclidean distance between the embedded test samples and all prototypical feature vectors.

The few-shot methods are claimed for the classification of datasets containing a small number of examples in each class. But their high accuracy (up to 96\%) [Ahmed] in experiments is achieved only on large datasets such as American Sign Language [Vaezi] and drops sharply with a decrease in the size of the dataset $(74.7 \%$ for Flemish Sign Language corpus [De Coster] and even less, up to 48,6\% [De Coster, Pigou]).

Thus, the problem of experimental evaluation of the effectiveness of various few-shot methods in relation to small-volume datasets, typical for medical practice, arises. To solve it, in our article we carried out:

- Improving few-shot techniques for practical application.

- $\quad$ Testing and analyzing few-shot techniques on SL recognition datasets.

\section{PROPOSED MODIFICATION AND MATERIAL USED}

Few-shot learning techniques discussed above are based on the concept of episodes, that is, they classify within a subset of classes, which allows the model to work more accurately. This limitation makes such an approach irrelevant for the problem of marker recognition of SLs. In this case, we need a method of online processing of the input stream of gestures, which, as a response, gives out a specific gesture from the entire alphabet of gestures, that is, makes a choice on the full set of classes (possible gestures), and not on a limited subset of classes considered as an episode. To do this, we propose a problem-oriented modification of the existing few-shot approach, detailed in the next section. Due to it, we propose a modification of the few-shot learning method shown in Figure 1 and described in detail below.

(A): Standard approach to teaching where we have access to training examples and information about to which class each sample belongs to. We train a model with an output dimension equal to the number of classes.

(B): Few-shot learning, this approach is using when there are a large number of classes and a small number of examples within each class. The dataset is divided into episodes, usually of 2-10 classes in each episode. Episode consist of examples (train) shots and queries (test) shots. Based on examples, the model "remembers" how each class looks like and, when receiving some queries (test) shots it is able to carry out a classification. The task of the machine learning model in this setting is to learn to distinguish different classes by remembering how each class in episode looks like.

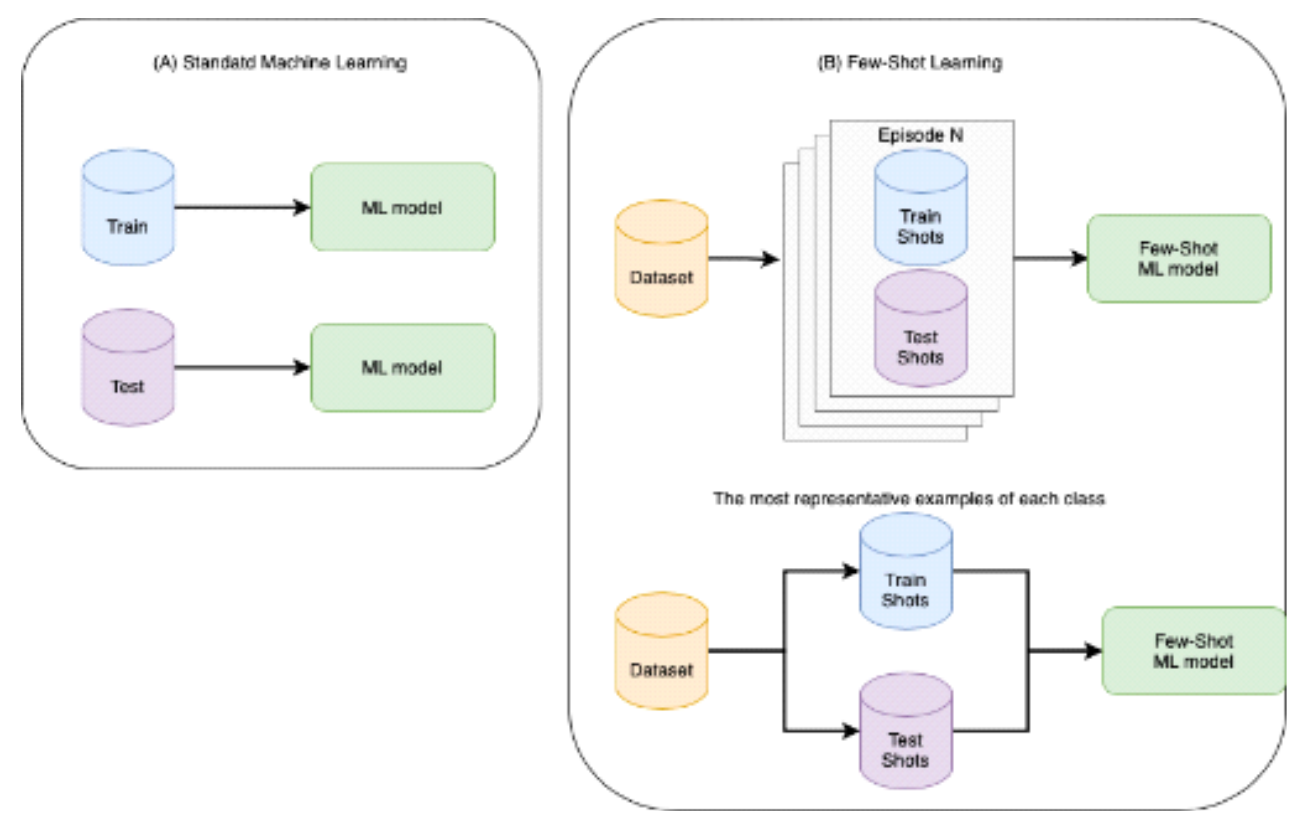

Figure 1. Examples of different approaches to model training 
(B) bottom image: our modification, where we do not use splitting into episodes, but select the most representative examples from the dataset and train the model to "remember" them, then when model receive the test shots model decides which class each sample belongs to.

In order to make a method work online we propose to put k-way equal to the full size of the alphabet, so in our settings we consider all evaluation as one big episode with a most representative samples chosen as examples of class. Thus, the pipeline for processing the input stream of gestures is as follows: (1) The number of classes in the episode is set equal to the number of classes in the dataset (2) We train the number of models using few-shot learning techniques (3) the best of trained models can be used in practice.

For investigations, we used a dataset for automatic SL recognition compiled from Surface Electromyography (EMG) and Inertial Measurement Unit (IMU) [Pacifici]. This dataset contains 26 gestures of the Italian SL alphabet. For each gesture, 30 data acquisitions were executed, composing a total of 780 samples included in the dataset. The gestures were performed by the same subject (male, 24 years old) in lab settings. EMG and IMU data were collected in a 2 seconds time window, at a sampling frequency of $200 \mathrm{~Hz}$.

\section{EXPERIMENTS}

To test the effectiveness of the proposed modification as applied to on-line processing of sign language, we have selected three models, covering all types of few-shot learning presented in Section 2, namely: Model-Agnostic Meta-Learning [Finn], Matching Networks [Vinyals], and Prototypical Networks [Snell]. The first one is optimization-based, the last two are metric-based. All models chosen are the most common in the field.

We used the following notation. In general, in standard machine learning each model is provided with a set of $\mathrm{m}$ ' labelled samples from 'C 'classes. Within few-shot learning 'C 'is traditionally called a ' $\mathrm{k}$-way', and ' $\mathrm{m}$ ' is called a ' $\mathrm{n}$-shot' inside each episode. In addition, to configure models, additional parameter $\mathrm{Q}$ is introduced - the number of examples per class. We evaluated the models by categorical efficiency (CA), i.e. the frequency of the correct class definition. We left all the parameters of the models architectures unchanged. The only modification was changing the size of the input space of neural networks, which was adjusted to our dataset and was equal to $400 * 8$. The experimental results are presented in Table 1 .

Table 1. Categorial accuracy for different few-shot learning models

\begin{tabular}{|c|c|c|l|l|l|l|l|l|l|c|}
\hline \multicolumn{4}{|c|}{ MAML [Finn] } & \multicolumn{3}{|c|}{ Matching Networks [Vinyals] } & \multicolumn{4}{c|}{ Prototypical Neworks [Snell] } \\
\hline k-way & n-shot & Q & CA & k-way & n-shot & CA & k-way & n-shot & Q & CA \\
\hline 5 & 3 & 1 & 0.66 & 3 & 3 & 0.919 & 26 & 1 & 1 & 0,655 \\
5 & 3 & 5 & 0.739 & 5 & 3 & 0.947 & 26 & 5 & 1 & 0,649 \\
5 & 3 & 7 & 0.993 & 8 & 3 & 0.807 & 26 & 13 & 1 & 0,653 \\
26 & 3 & 5 & 0,18 & 13 & 3 & 0.664 & 26 & 13 & 5 & 0,649 \\
26 & 3 & 7 & 0,21 & 26 & 3 & 0.549 & 26 & 13 & 10 & 0,647 \\
\hline
\end{tabular}

Comparison of the data in the table shows that with a small episode size ( $\mathrm{k}$-way $=1-5)$, the accuracy of all methods is quite high and corresponds to analogs. At the same time, if the episode size is chosen equal to the total number of classes in the dataset $(\mathrm{k}$-way $=26)$, then metric-based approaches still provide sufficient classification accuracy, comparable or even superior to analogs, while the accuracy of the optimization-based approach drops to unrealistic low values. Thus, our experiments provide a good basis for constructing classifiers for SL languages having extremely small datasets, as well as demonstrate the advantage of metric-based few-shot methods over optimization-based few-shot methods for solving the problem.

\section{CONCLUSION AND FUTURE WORK}

We investigated the possibility of classifying datasets with an extremely small number of samples of electromyograms of deaf and dumb gestures using few-shots learning methods. Several such methods have been considered - Matching Networks, Model-Agnostic Meta-Learning, and Prototypical Networks.

The developed methodology makes it possible to train electromyogram classifiers using an extremely small amount of data for other deaf and dumb sign languages. To do this, it is enough to collect a small dataset for re- or additional training of these models for the classification of another language, which is easy to accomplish 
in practice by means of a small session of recording the gestures of several deaf-mute people - speakers of a particular sign language.

Despite the results obtained, our research is still in the process of development, the accuracy achieved by the proposed methods can be significantly increased. First of all, we plan to use various augmentation techniques for this. The possibility of pre-training on data similar in distribution to the existing dataset is also being considered. In addition, in the future, it is planned to use active learning techniques to find the most representative samples from the dataset.

\section{ACKNOWLEDGEMENT}

This work was financially supported by Russian Science Foundation, Grant 19-19-00696.

\section{REFERENCES}

Agrawal S.C., Jalal A.S., Tripathi R.K. A survey on manual and non-manual sign language recognition for isolated and continuous sign, Int. J. Appl. Pattern Recognit., vol. 3, no. 2, pp. 99-134, 2016.

Ahmed M.A., Zaidan B.B. et al. A Review on Systems-Based Sensory Gloves for Sign Language Recognition State of the Art between 2007 and 2017. Sensors (Basel). 2018 Jul; 18(7): 2208.

Bragg D., Koller O., Bellard M. et al. Sign Language Recognition, Generation, and Translation: An Interdisciplinary Perspective. arXiv:1908.08597v1 [cs.CV] 22 Aug 2019.

De Coster M., Van Herreweghe M., Dambre J. Sign Language Recognition with Transformer Networks. Proc. 12th Conf. on Language Resources and Evaluation (LREC 2020), pp.6018-6024.

Ee L.W.S., Ramachandiran C.R., Logeswaran R. Real-Time Sign Language Learning System. J. Phys.: Conf. Ser. 1712 (2020) 012011.

Finn C., Abbeel P., Levine S., 2017. Model-agnostic meta-learning for fast adaptation of deep networks, PMLR, Int.Conv. Centre, Australia. pp. 1126-1135. http://proceedings. mlr.press/v70/finn17a.html.

Guo D., Zhou W., Li H., Wang M. Hierarchical LSTM for sign language translation, in Proc. 32nd AAAI Conf. Artif. Intell., Apr. 2018, pp. 6845-6852.

Huang J., Zhou W., Zhang Q., et al. Video-based sign language recognition without temporal segmentation, in Proc. 32nd AAAI Conf. Artif. Intell., Apr. 2018, pp. 1-8

Kamal S.M., Chen Y., Li S., et al. Technical Approaches to Chinese Sign Language Processing: A Review. IEEE Access, V. 7, 2019. DOI 10.1109/ACCESS.2019.2929174.

Koch G., Zemel R., Salakhutdinov R., 2015. Siamese neural net- works for one-shot image recognition, in: ICML deep learning work- shop, Lille.

Lee Y., Choi S., Gradient-based meta-learning with learned layerwise metric and subspace, in: International Conference on Machine Learning. 2018

Myo SDK Beta 7. https://developerblog.myo.com/myo-sdk-beta-7/

O'Connor T.F., et al. The Language of Glove: Wireless gesture decoder with low-power and stretchable hybrid electronics. PLoS ONE 12(7): e0179766. https://doi.org/10.1371/journal.pone.0179766

Pacifici I., Sernani P., Falcionelli N., et al. A surface electromyography and inertial measurement unit dataset for the Italian Sign Language alphabet https://www.ncbi.nlm.nih.gov/pmc/articles/PMC7644570/

Pigou L., Van Herreweghe, M., Dambre J. Sign classification in sign language corpora with deep neural networks. Int.Conference on Language Resources and Evaluation (LREC), Workshop, 2016, pp. 175-178

Snell J., Swersky K., Zemel R., 2017. Prototypical networks for few-shot learning, in: Advances in neural information processing systems, pp. 4077-4087.

Song Y., Lee S., Choi Y., et al. Design framework for a seamless smart glove using a digital knitting system. Fash Text 8 , 6 (2021). https://doi.org/10.1186/s40691-020-00237-2

Vaezi Joze H.R., Koller O. MS-ASL: A Large-Scale Data Set and Benchmark for Understanding American Sign Language. https://arxiv.org/pdf/1812.01053.pdf

Vinyals O., Blundell C., Lillicrap T., Kavukcuoglu K., Wierstra D. Matching Networks for One Shot Learning. arXiv:1606.04080v2 [cs.LG] 29 Dec 2017

Wang Y., Yao Q. , Kwok J., Ni L.M. Generalizing from a Few Examples: A Survey on Few-Shot Learning., https://arxiv.org/abs/1904.05046

Zhou Z., Chen K., Li X., et al. Sign-to-speech translation using machine-learning-assisted stretchable sensor arrays. Nat Electron 3, 571-578 (2020). https://doi.org/10.1038/s41928-020-0428-6 